\title{
Was bedeutet wissenschaftliche Qualität von Politikberatung?
}

\author{
Die Qualität wissenschaftlicher Politik- \\ beratung ist Dauerthema geworden. \\ Die Gründe liegen nahe. Politik benötigt \\ wissenschaftliches Wissen. Wissenschaft \\ wird politisch häufig nur dann unterstützt, \\ wenn sie ihren gesellschaftlichen Nutzen \\ demonstriert. \\ Von Reinhard F. Hüttl und Andreas Stucke
}

$\mathbf{P}$ litik und Wissenschaft sind zur Erfüllung ihrer jeweiligen Aufgaben unmittelbar aufeinander angewiesen (vgl. ausführlich und systematisch dazu Weingart 2001). Dabei müssen beide Seiten darauf achten, auch wenn sie sich immer häufiger auf gemeinsamen Foren begegnen, dass sie ihrer jeweils eigenen Handlungslogik folgen: die Politik also vorrangig Entscheidungen trifft und sich nicht in unendlichen Reflexionen über die Grundlagen ihres Tuns verliert und die Wissenschaft neues Wissen produziert und nicht hauptsächlich vorhandenes Wissen für eine wechselnde Tagespolitik aufbereitet.

Diese einfache Erkenntnis hilft, unrealistische Erwartungen zwischen Politikern und Wissenschaftlern zu vermeiden und der Versuchung nachzugeben, das Problem der Qualität von Politikberatung normativ durch Politiker- oder Wissenschaftlerschelte lösen zu wollen.

\section{Politikberatung ist Interaktionsprodukt}

Wissenschaftliche Politikberatung ist ein Interaktionsprodukt, dessen Qualität von notwendigen Voraussetzungen in beiden Teilbereichen sowie einem überzeugenden Brückenkonzept zur Verbindung beider Teilbereiche abhängt. In nuce: Gute wissenschaftliche Politikberatung bedarf auf der Seite der Wissenschaft vor allem methodisch gesichertes neues Wissen, das sich prinzipiell auf drängende gesellschaftliche Problemlagen beziehen lässt. Aufseiten der Abnehmer des Wissens, der Politik, erfordert gelungene wissenschaftliche Politikberatung die Bereitschaft und Fähigkeit, dieses Wissen reflexiv zu verarbeiten und umzusetzen.

Und schließlich bedarf es geeigneter Gelegenheiten und Organisationsformen, bei denen sich Akteure aus beiden Sphären begegnen und über Erwartungen und Regeln ihrer Zusammenarbeit und die Übersetzung praktischer Probleme in wissenschaftliche Fragestellungen und wissenschaftlicher Ergebnisse in anwendbare Handlungsempfehlungen verständigen. Die wissenschaftliche Qualität des bereitgestellten Wissens kann also noch keine ausreichende Voraussetzung für erfolgreiche Politikberatung sein; sie ist aber eine notwendige Bedingung und durch politisch-normatives Handlungswissen sowie praktischen Erfolg als Qualitätsmaßstab nicht ersetzbar. Das klingt wie ein Truismus. Dennoch treten gerade hier immer wieder Spannungen zwischen Wissenschaft und Politik auf, wie im Folgenden an zwei Beispielen gezeigt wird.

\section{Wissenschaftliche Qualität: Grundlage guter Politikberatung}

Eine erfolgreiche Wirtschaftspolitik gilt als Schlüssel für Wohlstand, Lebensqualität und Entwicklung. Alle modernen Volkswirtschaften haben deshalb in unterschiedlichen institutionellen Formen und Ausprägungen wirtschaftswissenschaftliche Beratungsprozesse als Grundlage wirtschaftspolitischen Entscheidens organisiert. Ob dies auf Bundesebene, wie in den USA, durch einen Council of Economic Advisers geschieht, der den jeweiligen Präsidenten berät, durch ein temporäres universitäres Netzwerk von Wirtschaftswissenschaftlern, wie in Großbritannien, oder große außeruniversitäre Forschungsinstitute, wie in Deutschland, hat für die Frage der wissenschaftlichen Qualität dieser Beratung keine erkennbare Bedeutung. Wissenschaftliche Qualität bedeutet in dem einen wie dem anderen System: Eigene qualifizierte Forschungsleistungen in Form von anerkannten, insbesondere empirischen Publikationen, sichergestellt durch Verfahren der internen und externen Qualitätskontrolle und indiziert durch Erfolge im Wettbewerb um hoch bewertete Drittmittel.

Um diese Kriterien hat es vor einigen Jahren heftige Debatten in Deutschland gegeben. Auslöser war der Wissenschaftsrat, der die großen Wirtschaftsforschungsinstitute evaluiert hatte und feststellte, dass in etlichen Einrichtungen die Qualität der wissenschaftlichen Fundierung nicht in ausreichendem Maße gegeben war (WR 1998). Für den Wissenschaftsrat war klar: Die Politik kann auf diese Weise nicht optimal beraten werden. Dem widersprach das Bundeswirtschaftsministerium als damaliger Auftraggeber der Institute mit einem Argument, das prototypisch für die Auseinandersetzung um gute Politikberatung ist.

Dieses Argument lautet, dass wissenschaftliche Exzellenz und auf Anwendung zielende Beratung zeitlich und sachlich miteinander konkurrieren, so dass Einrichtungen mit Beratungsaufgaben nur sehr beschränkt wissenschaftlichen Ex- $\rightarrow$ 


\section{„Gelungene Politikberatung, etwa für Nachhaltigkeit im Agrarsektor, bedarf mehr als nur qualifizierter agrarökonomischer Forschung. "}

zellenzkriterien genügen können aber auch nicht müssen. Es reiche vielmehr aus, wenn diese Einrichtungen hauptsächlich vorhandenes Wissen aufbereiteten, sammelten und der Politik kurzfristig zur Verfügung stellten. Eigene Forschung der Einrichtungen, die sich als Beiträge in anerkannten Zeitschriften niederschlage, richte sich demgegenüber fast ausschließlich an die wissenschaftliche Fachgemeinschaft und sei für die Belange der Politik nachrangig, jedenfalls nicht besonders zu fördern.

\section{Offenheit des Wissens trotz Transformation}

Ein solches Argument verkennt zweierlei: Erstens besteht wissenschaftliche Qualität von Politikberatung wesentlich darin, praktische Fragestellungen nicht nur angemessen auf aktuell verfügbares Wissen zu beziehen, sondern dieses Wissen so für praktische Zwecke transformieren zu können, dass einerseits die Informationsbedürfnisse außerwissenschaftlicher Adressaten befriedigt werden und gleichzeitig dieses Wissen für den Erkenntnisfortschritt offen gehalten wird.

Ohne ein bestimmtes Maß an qualifizierter eigener Forschung ist es nicht möglich, dieses notwendige Spannungsverhältnis zwischen aktuellen Beratungsbedürfnissen und Offenhalten für den wissenschaftlichen Erkenntnisfortschritt kompetent zu bewältigen. Zweitens müssen die Adressaten wissenschaftlichen Beratungswissens ein eigenes Interesse daran haben, dass dieses Wissen nicht dadurch entwertet wird, dass es öffentlich als veraltet, methodisch fragwürdig oder verdeckt politisch beeinflusst wahrgenommen wird. Das betrifft alle Einrichtungen, deren Hauptaufgabe darin besteht, Wissen für staatliches Handeln bereitzustellen, also auch die gesamte so genannte Ressortforschung des Bundes und der Länder (1).

Das bedeutet nicht, dass andere Faktoren für den Erfolg der Politikberatung weniger wichtig sind. So gehört zur Transferqualität des bereitgestellten Handlungswissens, dass es problem- und adressatenbezogen aufbereitet und in öffentlichen Debatten erprobt wurde.

\section{Fehlende Relevanz der Fachwissenschaft}

Dass andererseits im Zuge der von den referierten wirtschaftswissenschaftlichen Fachzeitschriften häufig belohnten Spezialisierung und Mathematisierung der Forschung die praktische Relevanz und Anwendbarkeit dieses Wissens, etwa für die Arbeitsmarktpolitik, auch zum Problem werden kann, wird von Ökonomen inzwischen selbst angesprochen (Lepenies 1997). Dennoch: Gute wissenschaftliche Politikberatung kann es nur auf der Grundlage qualifizierter wissenschaftlicher Arbeit geben, die in der Regel durch Publikationen auch in anerkannten Zeitschriften dokumentiert ist.

In einem anderen gesellschaftlich wichtigen Zukunftsbereich, der wissenschaftlichen Beratung für eine Nachhaltige Entwicklung, war und ist die Frage der wissenschaftlichen Qualität der Forschung ebenfalls Gegenstand wissenschaftspolitischer Kontroversen. Hier begegnet man im Kontext von Evaluationen einem prototypischen Argument, das sich wie folgt charakterisieren lässt: Forschung für eine nachhaltige Entwicklung ist immer inter- und transdisziplinäre Forschung, die demzufolge nicht nach den Standards einzelner Disziplinen beurteilt werden kann. Da außerdem gesellschaftliche Akteure aktiv in diese Forschung einbezogen sind, wird hier der Begriff des "gemeinsamen Forschens mit gesellschaftlichen Anspruchsgruppen“ (Smerkar et al. 2005) verwendet, und die Forschung gesellschaftlich relevante Problemlagen direkt beeinflussen will, kann wissenschaftliche Exzellenz, gemessen an qualifizierten Publikationen, kein gültiger Qualitätsmaßstab für diese Forschung sein.

Ohne Zweifel: Gelungene Politikberatung, etwa für Nachhaltigkeit im Agrarsektor, bedarf mehr als nur qualifizierter agrarökonomischer oder ernährungswissenschaftlicher Forschung, die in referierten Journalen veröffentlicht wird. Aber ohne Verortung und ohne Anerkennung im Belohnungssystem der Wissenschaft besteht die Gefahr, dass eine solche Forschung Nischenforschung und damit letztlich wirkungslos bleibt.

\section{Evaluation politisch gewollter Forschung}

Dieses gilt umso mehr, als die manchmal aufgestellte Behauptung, es gebe keine hochrangigen Publikationsmöglichkeiten für interdisziplinäre Arbeiten, pauschal jedenfalls nicht zutrifft (Röbbecke et al. 2004). Entsprechend muss vor allem politisch gewollte und geförderte Forschung den Anschluss an das Wissenschaftssystem und vor allem an die Universitäten suchen und sich wissenschaftlichen Evaluation stellen, an der nicht nur die „believer“ teilnehmen. Ein Beispiel ist das laufende Förderprogramm „Sozial-ökologische Forschung“ des BMBF (2). Problemorientierte Forschung schadet ihrem eigenen Anliegen, wenn sie allgemeine Regeln wissenschaftlichen Arbeitens nicht nur außer Kraft setzt, sondern diesen Regeln ihre Bedeutung absprechen will.

Hier, und nicht primär an der Zahl der wissenschaftlichen Publikationen, setzte die Kritik des Wissenschaftsrates am Wuppertal-Institut an, die für öffentliche Kontroversen gesorgt hatte (WR 2002; zur Kritik an der Evaluation des Wissenschaftsrats vgl. z.B. Ziegler 2003). Dass ein Institut lediglich selektiv wissenschaftliche Ergebnisse, etwa der Klimaforschung, zur Kenntnis nimmt, um darauf seine politisch Empfehlungen aufzubauen, bleibt ein feststellbarer Qualitätsmangel dieser Art von Politikberatung. 


\section{„Ein Kodex \\ guter wissenschaftlicher \\ Politikberatung sollte \\ entwickelt werden."}

Grundsätzlich ist allen Nachhaltigkeitsforschern im Grenzgebiet zwischen verschiedenen Disziplinen zu raten, gegenüber den etablierten Disziplinen nicht nur die eigene Andersartigkeit - gewissermaßen in einer "splendid isolation“ - zu reklamieren, sondern sich aktiv an der Entwicklung ergänzender überprüfbarer Kriterien zu beteiligen, die sie für eine Evaluierung ihrer Arbeiten gelten lassen wollen. Positive Ansätze dazu sind bereits erkennbar (Bergmann et al. 2005).

\section{Qualitätssicherung von Politikberatung}

Um die wissenschaftliche Qualität von Politikberatung zu sichern, ist eine Reihe von Maßnahmen vorstellbar, die in Ansätzen bereits realisiert werden. Viele der Vorschläge finden sich in Empfehlungen des Wissenschaftsrates zu politikberatenden Einrichtungen und lassen sich im Kern dahingehend zusammenfassen, dass wissenschaftliche Politikberatung, die in Deutschland institutionell zum großen Teil außeruniversitär stattfindet, eng mit allen Teilbereichen des Wissenschaftssystems vernetzt sein sollte. Einrichtungen der Politikberatung sollten vor allem:

I eine regelmäßige interne und externe wissenschaftliche Qualitätskontrolle ihrer Arbeiten durchführen. Mehrheitlich mit Wissenschaftlern besetzte Beiräte aus allen Teilen des Wissenschaftssystems sollten die Arbeit der Einrichtungen begleiten. Dieses ist bis heute keine Selbstverständlichkeit.

I Es sollten Anreize für die Kooperation mit anderen Teilen des Wissenschaftssystems, insbesondere Hochschulen, gesetzt werden, um die bestmögliche Grundlage für gute Beratung zu erhalten.

I Die Ziele, Verfahren und Ergebnisse von Politikberatung sollten für die wissenschaftlichen Fachgemeinschaften transparent und damit wissenschaftlicher Kritik zugänglich sein. Es sollte eine Veröffentlichungspflicht für ganz oder überwiegend aus öffentlichen Mitteln finanzierte Gutachten und Forschungsberichte eingeführt werden.

Orientiert an den Kriterien Unabhängigkeit, Transparenz und Qualität von Beratung sollte außerdem ein Kodex guter wissenschaftlicher Politikberatung entwickelt werden, auf dessen Grundlage „Fehlverhalten“ öffentlich gemacht werden kann. Ein solcher breit akzeptierter Kodex fehlt bislang in Deutschland. Er könnte, ähnlich wie die Regeln guter wissenschaftlicher Praxis, die durch die Deutsche Forschungsgemein- schaft „gehütet“ werden, durch eine Institution mit einer hohen Reputation in Wissenschaft und Politik getragen werden, die damit als öffentliche „Wächterinstanz“ fungiert.

\section{Literatur}

Bergmann, M./ Brohmann, B./ Hoffmann, E./ Loibl, M. C./ Rehaag, R./ Schramm, E./ Voß, J.-P.: Qualitätskriterien transdisziplinärer Forschung. Ein Leitfaden für die formative Evaluation von Forschungsprojekten. Frankfurt/ M. 2005. Download: www.downloads.isoe.de

Lepenies, W.: Benimm und Erkenntnis. Über die notwendige Rückkehr der Werte in die Wissenschaften. Frankfurt/ M. 1997.

Röbbecke, M./ Simon, D./ Lengwiler, M./ Kraetsch, C.: Inter-Disziplinieren. Erfolgsbedingungen von Forschungskooperationen. Berlin 2004.

Smerkar, O./ Pohl, C./ Stoll-Kleemann, S.: Evaluation: Humanökologie und Nachhaltigkeitsforschung auf dem Prüfstand. In: GAIA 14,1, 2005, S. 73-76.

Weingart, P.: Die Stunde der Wahrheit. Zum Verhältnis der Wissenschaft zu Politik, Wirtschaft und Medien in der Wissensgesellschaft. Weilerswist 2001, S. 127-169.

WR - Wissenschaftsrat (Hrsg.): Stellungnahme zu Instituten der Blauen Liste. Wirtschaftsforschungsinstitute in den alten Ländern, Band III. Köln 1998

WR - Wissenschaftsrat (Hrsg.): Stellungnahme zum Wuppertal Institut für Klima, Umwelt, Energie (WI). (Drs. 5263/02), Köln 2002.

Ziegler, H.-V.: Wissenschaftsverständnis und Wissenschaftsrat. In: GAIA 12,2, 2003, S. 87-90.

\section{Anmerkungen}

(1) Entsprechend wurde der Wissenschaftsrat vom Deutschen Bundestag 2004 um eine Evaluation der Bundesressortforschung gebeten, wobei er im Rahmen einer „,aufgabenkritischen Überprüfung“ vor allem zur „Notwendigkeit eigenständiger wissenschaftlicher Forschung “ in den Einrichtungen und deren „wissenschaftlichen Qualität" Stellung nehmen soll. (Deutscher Bundestag Drs. 15/2665 vom 10.3. 2004).

(2) Der Evaluationsbericht zum BMBF-Förderprogramm „Sozial-ökologische Forschung “ betont deshalb vor allem die Notwendigkeit, die Fördermaß nahmen auf Effekte im Wissenschaftssystem, insbesondere an den Hochschulen, anzulegen (Bewertungsbericht zum Förderschwerpunkt „Sozial-ökologische Forschung“, Berlin 2005).

\section{AUTOREN + KONTAKT} an der BTU Cottbus. Er war von Januar 2003 bis zum Januar 2006 Vorsitzender der Wissenschaftlichen
Dr. Dr. hc. Reinhard F. Hüttl ist Leiter des Lehrstuhls für Bodenschutz und Rekultivierung Kommission des Wissenschaftsrates.

BTU Cottbus, Postfach 101344, Cottbus. Tel.0335/69-2117, E-Mail: huettl@tu-cottbus.de

Dr. Andreas Stucke ist Leiter des Arbeitsbereichs Ressortforschung in der Geschäftstelle des Wissenschaftsrates (WR). Geschäftstelle des WR, Brohler Str. 11, 50968 Köln. Tel. 0221/3776-257,

E-Mail: stucke@wissenschaftsrat.de

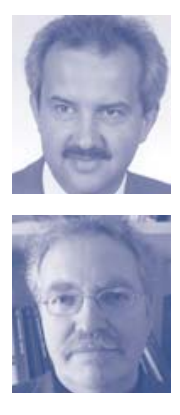


(c) 20I0 Authors; licensee IÖW and oekom verlag. This is an article distributed under the terms of the Creative Commons Attribution Non-Commercial No Derivates License (http://creativecommons.org/licenses/by-nc-nd/3.o/), which permits unrestricted use, distribution, and reproduction in any medium, provided the original work is properly cited. 